\title{
A Cost Analysis of Gyrase A Testing and Targeted Ciprofloxacin Therapy Versus Recommended 2-Drug Therapy for Neisseria gonorrhoeae Infection(Article)
}

- Allan-Blitz, L.-T.aEmail Author,

- Hemarajata, P. ${ }^{\mathrm{b}}$,

- Humphries, R.M. ${ }^{\text {, }}$

- Wynn, A.c,

- Segura, E.R. ${ }^{d}$,

- Klausner, J.D.ce

- View Correspondence (jump link)

- $\quad$ aDivision of Infectious Diseases, Department of Medicine, United States

- 'Department of Laboratory Medicine, David Geffen School of Medicine, Department of Medicine, United States

- 'Fielding School of Public Health, University of California Los Angeles, Los Angeles, CA, United States

View additional affiliations

Abstract_View references (27)

Background Novel approaches to combating drug-resistant Neisseria gonorrhoeae infections are urgently needed. Targeted therapy with ciprofloxacin has been made possible by a rapid assay for genotyping the gyrase A (gyrA) gene; a nonmutated gene reliably predicts susceptibility to ciprofloxacin. Methods We determined the costs of running the gyrA assay, $500 \mathrm{mg}$ of ciprofloxacin, $250 \mathrm{mg}$ of ceftriaxone injection, and $1000 \mathrm{mg}$ of azithromycin. Cost estimates for gyrA testing included assay reagents and labor. Cost estimates for ceftriaxone included medication, injection, administration, supplies, and equipment. We measured the cost of using the gyrA assay and treatment based on genotype using previously collected data over a 13-month period between November 2015 and November 2016 for all N. gonorrhoeae cases diagnosed at UCLA. We subsequently developed 3 cost models, varying the frequency of testing and prevalence of N. gonorrhoeae infections with ciprofloxacin-resistant or genotype-indeterminate results. We compared those estimates with the cost of recommended 2 -drug therapy (ceftriaxone and azithromycin). Results Based on a $65.3 \%$ prevalence of cases with ciprofloxacin-resistant or genotype indeterminate N. gonorrhoeae infections when running an average of 1.7 tests per day, the per-case cost of gyrA genotyping and targeted therapy was US \$197.19. The per-case cost was US \$155.16 assuming a 52.6\% prevalence of ciprofloxacin-resistant or genotype-indeterminate infections when running an average of 17 tests per day. The per-case cost of 2-drug therapy was US \$142.75. Conclusions Direct costs of gyrA genotyping and targeted ciprofloxacin therapy depend on the prevalence of ciprofloxacin-resistant or genotype-indeterminate infections and testing frequency. (C) 2018 Lippincott Williams \& Wilkins.

Indexed keywords 
EMTREE drug terms:

EMTREE medical terms:
azithromycinceftriaxoneciprofloxacinDNA topoisomerase (ATP hydrolysing) A

antibiotic resistanceantibiotic sensitivityArticlechlamydiasiscost benefit analysis assaygenotypegonorrheahumanmajor clinical studymixed infectionprevalence

Chemicals and CAS Registry Numbers:

azithromycin, 83905-01-5, 117772-70-0, 121470-24-4; ceftriaxone, 73384-59-5, 74578-69-1;

ciprofloxacin, 85721-33-1

Funding details

Funding number Funding sponsor

R21AI117256 Foundation for the National Institutes of Health

R21AI109005 Foundation for the National Institutes of Health

School of Medicine

University of California, Los Angeles

Facultad de Ciencias, Universidad de los Andes

Division of Intramural Research, National Institute of Allergy and Infectious Diseases

U.S. Department of the Interior

Department of Veterinary Medicine, Cambridge Veterinary School

R25MH087222

- Funding text

Department of Medicine, Ottawa Hospital

From the *Division of Infectious Diseases, Department of Medicine, $\dagger$ Department of Laboratory Medicine, David Geffen School of Medicine, Department of Medicine, tFielding School of Public Health, University of California Los Angeles, Los Angeles, CA; §Escuela de Medicina, Universidad Peruana de Ciencias Aplicadas, Lima, Peru; and IDDivision of Infectious Diseases, Department of Medicine, University of California Los Angeles, Los Angeles, CA Conflict of Interest and Sources of Funding: None declared. This research was supported by the United States National Institutes of Health grants R21AI117256 and R21AI109005 as well as the South American Program in HIV Prevention Research NIH/NIMH R25MH087222. Correspondence: Lao-Tzu Allan-Blitz, MD Candidate, 2018, David Geffen School of Medicine, University of California Los Angeles, 10833 Le Conte Ave, Los Angeles, CA 90095. E-mail: lallanblitz@mednet.ucla.edu. Received for publication February 21, 2017, and accepted July 30, 2017. DOI: 10.1097/OLQ...View All

- ISSN: 01485717

- CODEN: STRDD

- Source Type: Journal

- Original language: English

- DOI: 10.1097/OLQ.0000000000000698

- Document Type: Article

- Publisher: Lippincott Williams and Wilkins 